\title{
NONOSCILLATION AND DISCONJUGACY IN THE COMPLEX DOMAIN
}

\author{
BY
}

\author{
PAUL R. BEESACK
}

0. Introduction. This paper $\left({ }^{1}\right)$ deals with the zeros of solutions of the second-order, linear differential equation

$$
w^{\prime \prime}(z)+p(z) w(z)=0,
$$

where $p(z)$ is a function analytic in a region $R$ of the complex plane. E. Hille $[3 ; 4]$ was the first to make a systematic study of the distribution of the zeros of solutions of (0.1). His approach consisted of selecting a particular zero $z=a$ of a particular solution $w(z)$ of $(0.1)$, and then constructing a zero-free region about $z=a$, i.e., a region about $a$ in which $w(z)$ does not vanish again. Further mention of this is made in $\$ 1$; additional methods of constructing such zero-free regions are also given in $\$ \$ 3,7$, and 9.

More recently, $Z$. Nehari $[7 ; 9 ; 10]$ made use of a relationship between the theory of univalent functions and the theory of equations of the above type to obtain results of quite a different nature. This relationship is provided by the Schwarzian derivative

$$
\{f(z), z\}=\left[f^{\prime \prime}(z) / f^{\prime}(z)\right]^{\prime}-\left[f^{\prime \prime}(z) / f^{\prime}(z)\right]^{2} / 2
$$

of a function $f(z)$ analytic in a region $R$. Indeed the univalence of $f(z)$ in $R$ is equivalent to the fact that no solution of

$$
w^{\prime \prime}(z)+\{f(z), z\} w(z) / 2=0
$$

has more than one zero in $R$. The above relationship provides a kind of duality principle for many of the results of this paper. All of the results are stated as theorems about differential equations, and the dual results concerning univalence (or $n$-valence) are only mentioned occasionally.

Following the usage of Wintner [17] in the real case, and of Taam [14] in the complex case, we say that equation $(0.1)$ is disconjugate on a point set $S$ of the complex plane if no solution of $(0.1)$ vanishes more than once in $S$. The equation is said to be nonoscillatory in $S$ if none of its solutions vanishes

Received by the editors June 23, 1955.

(1) This paper forms part of the author's thesis accepted in partial fulfillment of the requirements for the degree Ph.D. at Washington University in January, 1955.

This research was supported by the United States Air Force, through the Office of Scientific Research of the Air Research and Development Command. 
more than a finite number of times in $S$. (Thus disconjugacy corresponds to univalence, and nonoscillation to finite valence.)

The main results concerning disconjugacy are in $\$ \S 2,4$, and 6 . These results are of a similar character to those in $[7 ; 9 ; 10]$, and are in the form of sufficient conditions that $(0.1)$ be disconjugate in a disk. In each case the problem is reduced to an examination of a real differential equation (usually involving a parameter). The results are closely related, not only to those of Nehari, but also to some criteria of V. V. Pokornyi [11]. Indeed, using some lemmas on real differential equations (some of which were announced by Pokornyi) proved in $\$ 5$, we show that Pokornyi's criteria are contained in a general theorem of $\$ 4$ which gives a lower bound for the distance between zeros of any solution of $(0.1)$. In $\S 3$ we investigate the distribution of zeros of solutions of $(0.1)$ in a neighborhood of a regular singular point of (0.1). By means of the Green's function, introduced in $\S 7$, equation ( 0.1$)$ is transformed into an integral equation. This section provides the basis for the nonoscillation theorems of $\S 8$, and for the criteria of 3-valence and disconjugacy of $\S \S 9$ and 10.

The author wishes to thank Professor Zeev Nehari for his generous help and guidance in the preparation of this paper.

1. A comparison theorem. We begin this section with the following elementary lemma involving real functions.

LEMмA 1.1. Let $f(x)$ be continuous on the interval $a<x<b$, with

$$
f(x)=O\left[(x-a)^{-2}\right] \text { as } x \rightarrow a+, \quad f(x)=O\left[(b-x)^{-2}\right] \text { as } x \rightarrow b-.
$$

Moreover, suppose the Riccati equation

$$
g^{\prime}=f(x)+g^{2}
$$

has a solution $g(x)$ of class $C^{1}$ on $a<x<b$, with

$$
g(x)=O\left[(x-a)^{-1}\right] \text { as } x \rightarrow a+, g(x)=O\left[(b-x)^{-1}\right] \text { as } x \rightarrow b-.
$$

If $y(x)$ is piecewise smooth on $a \leqq x \leqq b$, and if $y(a)=y(b)=0$, then

$$
\int_{a}^{b}\left(y^{\prime 2}-f y^{2}\right) d x=\int_{a}^{b}\left(y^{\prime}+g y\right)^{2} d x .
$$

This is easily proved by expanding the right side of (1.1) and carrying out an appropriate integration by parts. Our interest in (1.1) is due to the fact that it asserts that the integral on the left side is non-negative. For special cases of this lemma see [1, Theorems 257, 262], and [7].

We note that if the differential equation $Y^{\prime \prime}+f(x) Y=0$ has a solution $Y(x) \neq 0$ on $a<x<b$, then the above Riccati equation has the solution $g(x)=-Y^{\prime}(x) / Y(x)$ satisfying the above conditions. Moreover, if $Y(x) \neq 0$ on $a \leqq x \leqq b$, then, provided $y(x) \not \equiv 0$, the right side of (1.1) is strictly positive. 
Now, let $p(z)$ be an analytic function of the complex variable $z$ in a simplyconnected region $D$. Consider the differential equation

$$
w^{\prime \prime}+p(z) w=0 .
$$

Let $w(z)$ be a solution of this equation; then $w(z)$ is also analytic in $D$. Multiply both sides of (1.2) by

$$
\overline{w(z)}
$$

and integrate along any path in $D$ joining two points $z=a, z=b$, to obtain

$$
\int_{a}^{b} w^{\prime \prime} \bar{w} d z+\int_{a}^{b} p|w|^{2} d z=0 .
$$

Integrating by parts the first term we obtain

$$
\left.\overline{w(z)} w^{\prime}(z)\right|_{a} ^{b}-\int_{a}^{b}\left|w^{\prime}\right|^{2} \overline{d z}+\int_{a}^{b} p|w|^{2} d z=0 .
$$

This expression is called the Green's transform of equation (1.2) and is due to Hille $[3 ; 4]$.

We now specialize the Green's transform to the case that $w(a)=w(b)=0$, and the path is taken to be the straight line $z=a+r e^{i \theta}$ joining $a$ and $b$. We obtain

$$
\int_{0}^{R}\left|w^{\prime}\right|^{2} d r=e^{2 i \theta} \int_{0}^{R} p|w|^{2} d r, \quad R=|b-a| .
$$

Setting $e^{2 i \theta} p\left(a+r e^{i \theta}\right)=q_{1}(r ; \theta)+i q_{2}(r ; \theta)$, the last equation is equivalent to the pair of equations

$$
\begin{aligned}
& \int_{0}^{R} q_{1}|w|^{2} d r=\int_{0}^{R}\left|w^{\prime}\right|^{2} d r, \\
& \int_{0}^{R} q_{2}|w|^{2} d r=0 .
\end{aligned}
$$

We shall now recombine equations (1.4), (1.5) by multiplying the first equation by a parameter $\lambda$, the second by a parameter $\mu$, and adding, to obtain

$$
\int_{0}^{R}\left(\lambda q_{1}+\mu q_{2}\right)|w|^{2} d r=\lambda \int_{0}^{R}\left|w^{\prime}\right|^{2} d r .
$$

We now use this modified Green's transform together with Lemma 1.1 to prove a comparison theorem.

THEOREM 1.1. Let $p(z)$ be analytic in a region $D$, and let the line $z=a+r e^{i \theta}$, $0 \leqq r \leqq R$, lie in $D$. Set 


$$
e^{2 i \theta} p\left(a+r e^{i \theta}\right)=q_{1}(r ; \theta)+i q_{2}(r ; \theta) .
$$

Let $Q(r)$ be continuous on $0 \leqq r \leqq R$, and suppose the differential equation

$$
y^{\prime \prime}+Q(r) y=0
$$

has a (real) solution $y(r)$ which does not vanish on $0<r<R$. If there exist real numbers $\lambda \geqq 0, \mu,\left(\lambda^{2}+\mu^{2} \neq 0\right)$, such that

$$
\lambda q_{1}(r ; \theta)+\mu q_{2}(r ; \theta) \leqq \lambda Q(r), \quad 0 \leqq r \leqq R,
$$

then any nontrivial solution $w(z)$ of $w^{\prime \prime}+p(z) w=0$ having $w(a)=0$ has no other zeros on the open line segment $\left(a, a+R e^{i \theta}\right)$ unless $q_{2}(r ; \theta) \equiv 0$. Even if $q_{2}(r ; \theta) \equiv 0$, the conclusion holds provided $\lambda \neq 0$. Moreover, if strict inequality holds in (1.8) for a single point, then $w(z)$ has no zeros on the half-closed segment $\left(a, a+R e^{i \theta}\right]$.

Proof. Assume $w(z)$ has a second zero at $b=a+R_{1} e^{i \theta}, R_{1}<R$. If $Q(r) \equiv 0$, $\lambda>0$, then (1.8) and (1.6) are incompatible. If $Q(r) \equiv 0, \lambda=0, \mu \neq 0$, then (1.8) and (1.5) are incompatible, unless $q_{2} \equiv 0$.

Now suppose $Q(r) \not \equiv 0$ on $0 \leqq r \leqq R_{1}$. Again we see that $\lambda=0$ leads to a contradiction, so that we need only consider the case $\lambda>0$. In this case, from (1.8) and (1.6) we obtain

$$
\int_{0}^{R_{1}}\left|w^{\prime}\right|^{2} d r \leqq \int_{0}^{R_{1}} Q(r)|w|^{2} d r .
$$

Now, let $\left|w\left(a+r e^{i \theta}\right)\right|=W(r)$. If $z=a+r e^{i \theta}$, then by applying Cauchy's inequality we see that $\left|W^{\prime}(r)\right| \leqq\left|w^{\prime}(z)\right|$, so that

$$
\int_{0}^{R_{1}} W^{\prime 2}(r) d r \leqq \int_{0}^{R_{1}} Q(r) W^{2}(r) d r .
$$

Let $y(r)$ be a nontrivial solution of (1.7) for which $y(0)=0$. Then $y(r)$ does not vanish on the interval $0<r<R$. Consequently, if $x(r)$ is a nontrivial solution of (1.7) for which $x\left(R^{\prime}\right)=0, R_{1}<R^{\prime}<R$, then $x(r)$ does not vanish on the interval $0 \leqq r \leqq R_{1}$, by the Sturm separation theorem. By the remark following Lemma 1.1 it follows that

$$
\int_{0}^{R_{1}} y^{\prime 2}(r) d r>\int_{0}^{R_{1}} Q(r) y^{2}(r) d r
$$

for every function $y(r) \not \equiv 0$, piecewise smooth on $0 \leqq r \leqq R_{1}$, and such that $y(0)=y\left(R_{1}\right)=0 . W(r)$ is such a function; but then we have a contradiction of (1.9), and the theorem is proved in the case $R_{1}<R$.

Finally, suppose (1.8) is a strict inequality for at least one point, hence also for an interval, of $0 \leqq r \leqq R$, and assume $b=a+R e^{i \theta}$ is a second zero of $w(z)$. The proof for the case $Q(r) \equiv 0$ is seen to hold for $R_{1}=R$ as does that for the case $Q(r) \not \equiv 0, \lambda=0$. In the general case $Q(r) \not \equiv 0, \lambda>0$ the inequality (1.9) 
becomes a strict inequality, and the result again follows using the remark following Lemma 1.1. (Note that the right side of (1.1) may be zero in this case.)

A theorem substantially equivalent to Theorem 1.1 has been proved, along essentially different lines, by C. T. Taam [14, Theorem 9].

Special cases of this theorem which are of interest are obtained for

(i) $Q(r)=\pi^{2} / R^{2}$;

(ii) $\lambda=1, \mu=0, Q \equiv q_{1}$; this case was proved by Taam [13], and also stated (incorrectly) by Pokornyi [11];

(iii) $\lambda=0, q_{2} \neq 0$ on $0 \leqq r \leqq R, \mu=-\operatorname{sgn} q_{2}$;

(iv) $\lambda=1, \mu=0, Q \equiv q_{1} \leqq 0$.

The last two cases were proved by Hille [3], and form the basis for the construction of his "zero-free stars." The first case gives us a result which was first proved by Nehari [7].

Corollary 1.1. Suppose that $p(z)$ is analytic in $|z|<R$, and that $|p(z)|$ $\leqq \pi^{2} / 4 R^{2},|z|<R$. Then no solution of $w^{\prime \prime}+p(z) w=0$ has more than one zero in $|z|<R$.

For, 1. $q_{1}(r ; \theta) \leqq\left|e^{2 i \theta} p\left(a+r e^{i \theta}\right)\right| \leqq \pi^{2} / 4 R^{2}$, so that the result follows from the special case (i), with $\lambda=1, \mu=0$.

Similarly, we may state the following corollary which will be of use in the sequel.

COROLlaRy 1.2. If the differential equation

$$
y^{\prime \prime}+\left|p\left(a+r e^{i \theta}\right)\right| y=0
$$

has a solution $y(r)$ which does not vanish on the interval $0<r<R$, then a nontrivial solution $w(z)$ of $w^{\prime \prime}+p(z) w=0$ having $w(a)=0$ does not vanish again on the segment $\left(a, a+R e^{i \theta}\right)$.

2. Sufficient conditions for disconjugacy in a circle. The proof of the following theorem is based on some of the ideas used in [9].

TheOREM 2.1. Let $p(z)$ be analytic in $|z|<\beta_{0} \leqq 1$. Set

$$
M(r)=\max _{|z|=r}|p(z)|, \quad 0 \leqq r<\beta_{0} ; M(-r)=M(r) .
$$

Suppose that for each $\beta, 0 \leqq \beta<\beta_{0}$, the differential equation

$$
\frac{d^{2} u}{d w^{2}}+\left(\frac{1-\beta^{2}}{1+\beta^{2} w^{2}}\right)^{2} M\left[\left(\frac{\beta^{2}+w^{2}}{1+\beta^{2} w^{2}}\right)^{1 / 2}\right] u=0
$$

has a (real) solution which does not vanish on the interval

$$
-\left(\frac{\beta_{0}^{2}-\beta^{2}}{1-\beta_{0}^{2} \beta^{2}}\right)^{1 / 2}<w<\left(\frac{\beta_{0}^{2}-\beta^{2}}{1-\beta_{0}^{2} \beta^{2}}\right)^{1 / 2} .
$$


Then the differential equation

$$
y^{\prime \prime}+p(z) y=0
$$

is disconjugate in $|z|<\beta_{0}$.

Proof. Assume that a solution $y(z)$ of (1.2) has two zeros, $z=a, z=b$, in $|z|<\beta_{0}$. There exists a unique circle which passes through these two points and is orthogonal to the circle $|z|=1$. By a rotation $\zeta=\alpha z,|\alpha|=1$, we may bring the arc of this circle which is contained in $|z| \leqq 1$ into a position in the upper half-plane so that it is symmetric with respect to the imaginary axis. The differential equation (1.2) is transformed into the equation

$$
y^{\prime \prime}+\alpha^{-2} p(\zeta / \alpha) y=0
$$

having the same modulus function $M(r)$. Hence there is no loss of generality in assuming the original orthogonal arc to be in this position. This arc cuts the imaginary axis in a point $z=i \beta$, with $\beta<\beta_{0}$, and the linear transformation $w=(z-i \beta) /(1+i \beta z)$ of $|z|<1$ onto $|w|<1$ carries the orthogonal arc onto the line segment $-1<w<1$. The points of intersection of the orthogonal arc with the circle $|z|=\beta_{0}$ are mapped into the points $w= \pm\left(\beta_{0}^{2}-\beta^{2}\right)^{1 / 2}\left(1-\beta_{0}^{2} \beta^{2}\right)^{-1 / 2}$ $= \pm w_{1}$, and the zeros $z=a, z=b$ are mapped into points on the interval $-w_{1}<w<w_{1}$.

At the same time, the linear transformation transforms the differential equation into the equation

$$
Y^{\prime \prime}-\frac{2 i \beta}{1-i \beta w} Y^{\prime}+\frac{\left(1-\beta^{2}\right)^{2}}{(1-i \beta w)^{4}} p\left(\frac{w+i \beta}{1-i \beta w}\right) Y=0,
$$

where $Y(w)=y(z)$. Now, if we let $u(w)=(1-i \beta w) Y(w)$, then $u(w)$ also has two zeros on the interval $-w_{1}<w<w_{1}$. The function $u(w)$ satisfies the differential equation

$$
\frac{d^{2} u}{d w^{2}}+\frac{\left(1-\beta^{2}\right)^{2}}{(1-i \beta w)^{4}} p\left(\frac{w+i \beta}{1-i \beta w}\right) u=0 .
$$

However, for real $w$, we have

$$
|1-i \beta w|^{4}=\left(1+\beta^{2} w^{2}\right)^{2}, \quad\left|\frac{w+i \beta}{1-i \beta w}\right|=\left(\frac{w^{2}+\beta^{2}}{1+\beta^{2} w^{2}}\right)^{1 / 2}
$$

hence by Corollary 1.2, equation (2.1) must have a solution which vanishes twice on the interval $-w_{1}<w<w_{1}$. But this is impossible since then, by the Sturm separation theorem, every solution of (2.1) would have at least one zero on this interval, contrary to hypothesis. In case $\beta_{0}=1, w_{1}$ must be replaced by 1 .

This criterion is closely related to that of $Z$. Nehari mentioned at the be- 
ginning of this section. The relationship will be established by noting that the conditions

$$
\begin{aligned}
& \left(\frac{1-\beta^{2}}{1+\beta^{2} w^{2}}\right)^{2} M\left[\left(\frac{\beta^{2}+w^{2}}{1+\beta^{2} w^{2}}\right)^{1 / 2}\right] \leqq M(w), \\
& 0 \leqq \beta<\beta_{0}, 0 \leqq w<\left(\frac{\beta_{0}^{2}-\beta^{2}}{1-\beta_{0}^{2} \beta^{2}}\right)^{1 / 2}
\end{aligned}
$$

and

$$
\left(1-r^{2}\right)^{2} M(r) \text { is nonincreasing on } 0 \leqq r<\beta_{0}
$$

are actually equivalent. To prove this, assume first that $(2.4)$ holds. Let $\beta$, $w$ be given subject to the conditions in (2.3). Set $x^{2}=\left(\beta^{2}+w^{2}\right) /\left(1+\beta^{2} w^{2}\right)$; then $w^{2} \leqq x^{2} \leqq \beta_{0}^{2}$, hence $\left(1-x^{2}\right)^{2} M(x) \leqq\left(1-w^{2}\right)^{2} M(w)$, which reduces to $(2.3)$. Conversely, suppose (2.3) holds. Let $w, r$ be given such that $0 \leqq w \leqq r<\beta_{0}$. If $\beta^{2}$ is defined by the equation $r^{2}=\left(\beta^{2}+w^{2}\right) /\left(1+\beta^{2} w^{2}\right)$, then it is easily seen that $\beta, w$ satisfy the conditions in (2.3). The inequality (2.3) now reduces to $\left(1-r^{2}\right)^{2} M(r) \leqq\left(1-w^{2}\right)^{2} M(w)$, establishing (2.4).

In view of this result, an application of the Sturm comparison theorem gives us

Corollary 2.1. Equation (1.2) is disconjugate in $|z|<\beta_{0}$ if $\left(1-r^{2}\right)^{2} M(r)$ is nonincreasing for $0 \leqq r<\beta_{0}$, and if the differential equation

$$
y^{\prime \prime}+M(r) y=0
$$

has a solution which does not vanish on the interval $-\beta_{0}<r<\beta_{0}$.

For $\beta_{0}=1$ this is equivalent to the criterion derived in [9]. As pointed out there, this result includes at least three sharp cases (for $\left.\beta_{0}=1\right)$, namely the cases $M(r)=\pi^{2} / 4, M(r)=2 /\left(1-r^{2}\right)$, and $M(r)=1 /\left(1-r^{2}\right)^{2}$. The first and last of these cases were originally proved in an earlier theorem of Nehari [7]; the remaining case is contained in a theorem announced (without proof) by V. V. Pokornyi [11]. As a matter of fact, the result contains the entire theorem of Pokornyi. Stated as a criterion of disconjugacy, this theorem states that if

$$
M(r) \leqq \begin{cases}2^{3 \lambda-2} \pi^{2(1-\lambda)} /\left(1-r^{2}\right)^{\lambda} & (0 \leqq \lambda \leqq 1), \\ 2^{2-\lambda} /\left(1-r^{2}\right)^{\lambda} & (1 \leqq \lambda \leqq 2),\end{cases}
$$

for $0 \leqq r<1$, then $w^{\prime \prime}+p(z) w=0$ is disconjugate in $|z|<1$.

In both cases we have $\left(1-r^{2}\right)^{2} M(r)$ nonincreasing on $0 \leqq r<1$, so that it suffices to find a Sturm majorant for equation (2.5). In the case $0 \leqq \lambda \leqq 1$, such a majorant is found in the equation $y^{\prime \prime}+f(r) y=0$, where

$$
f(r)=\frac{\pi^{2}}{4}(1-\lambda)+\frac{2 \lambda}{1-r^{2}}+\lambda(\cdot 1-\lambda)\left\{\frac{\pi}{2} \tan \frac{\pi r}{2}-\frac{2 r}{1-r^{2}}\right\}^{2} .
$$


This equation has the solution

$$
y(r)=\left(1-r^{2}\right)^{\lambda}(\cos \pi r / 2)^{1-\lambda}
$$

which is not zero on $-1<r<1$; the result relating to (2.6) now follows, for $0 \leqq \lambda \leqq 1$, from the fact that

$$
\frac{2^{3 \lambda-2} \pi^{2(1-\lambda)}}{\left(1-r^{2}\right)^{\lambda}} \leqq \frac{\pi^{2}}{4}(1-\lambda)+\frac{2 \lambda}{1-r^{2}}, \quad 0 \leqq r<1 .
$$

In the case $1 \leqq \lambda \leqq 2$, the equation $y^{\prime \prime}+g(r) y=0$, where

$$
g(r)=(3-\lambda) \frac{1-(2-\lambda) r^{2}}{\left(1-r^{2}\right)^{2}}
$$

is a suitable Sturm majorant for (2.5). For, this equation has the solution $y(r)=\left(1-r^{2}\right)^{(3-\lambda) / 2} ;$ moreover, the constant $2^{2-\lambda}$ of $(2.6)$ can be improved to $3-\lambda$ in view of the easily established inequality

$$
\frac{3-\lambda}{\left(1-r^{2}\right)^{\lambda}} \leqq g(r), \quad 0 \leqq r<1 .
$$

As another application of Corollary 2.1, this time for the case $\beta_{0}<1$, suppose that

$$
M(r) \leqq \frac{1}{\left(1-r^{2}\right)^{2}}+\frac{\nu(\nu+1)}{1-r^{2}}=Q(r),
$$

where $\nu$ is an even positive integer. Then if $P_{\nu}(r)$ denotes the Legendre polynomial of degree $\nu$, and if $y(r)=\left(1-r^{2}\right)^{1 / 2} P_{\nu}(r)$, we have

$$
y^{\prime \prime}(r)+Q(r) y(r) \equiv 0 .
$$

Hence, by Corollary 2.1 , if $\beta_{0}$ is the least positive zero of $P_{\nu}(r)$, and $M(r)$ satisfies (2.9), then the differential equation $w^{\prime \prime}+p(z) w=0$ is disconjugate in $|z|<\beta_{0}$. Moreover, the constant $\nu(\nu+1)$ is obviously sharp for disconjugacy in this circle.

3. Zeros in the neighborhood of a regular singular point. We first note the following special case of Lemma 1.1. If

$$
g(x)=\lambda-1 / 2 x
$$

then

$$
g^{\prime}(x)-g^{2}(x)=\frac{1}{4 x^{2}}+\frac{\lambda}{x}-\lambda^{2}=f(x),
$$

and $f(x), g(x)$ satisfy the hypotheses of Lemma 1.1 on any interval $0 \leqq x \leqq b$. It follows that 


$$
\int_{0}^{b}\left\{y^{\prime 2}-\left(\frac{1}{4 x^{2}}+\frac{\lambda}{x}-\lambda^{2}\right) y^{2}\right\} d x=\int_{0}^{b}\left\{y^{\prime}+\left(\lambda-\frac{1}{2 x}\right) y\right\}^{2} d x
$$

whenever $y(x) \in C^{1}$ on $0 \leqq x \leqq b$ with $y(0)=y(b)=0$. Now the integral on the right side of this equality vanishes only if $y^{2}=c x e^{-2 \lambda x}$, hence for all such $y(x)$ as noted, other than $y(x) \equiv 0$, we have

$$
\int_{0}^{b}\left(\frac{1}{4 x^{2}}+\frac{\lambda}{x}-\lambda^{2}\right) y^{2} d x<\int_{0}^{b} y^{\prime 2} d x
$$

Now, let $D$ be any simply-connected convex region of the complex plane. Suppose that $p(z)$ is analytic in $D$ except possibly at $z=0$ (if $0 \in D$ ), and that, for a fixed $\lambda \geqq 0$,

$$
|p(z)| \leqq \frac{1}{\left.4 ! z\right|^{2}}+\frac{\lambda}{|z|}-\lambda^{2},
$$

for all $z \in D$. If $z=0$ is in $D, p(z)$ can have a pole of at most the second order at this point, and consequently has the expansion $\left({ }^{2}\right)$

$$
p(z)=a z^{-2}+b z^{-1}+Q(z),
$$$$
|a| \leqq 1 / 4
$$

where $Q(z)$ is analytic at $z=0$. The differential equation

$$
w^{\prime \prime}+p(z) w=0
$$

will then have $z=0$ as a regular singular point. Suppose that a nontrivial solution $w(z)$ of this equation has two zeros in $D$, say $w(a)=w(b)=0$. By (1.3) we have

$$
\int_{0}^{R}\left|w^{\prime}(z)\right|^{2} d r=e^{2 i \theta} \int_{0}^{R} p(z)|w(z)|^{2} d r
$$

where $z=a+r e^{i \theta}, b-a=R e^{i \theta}$, and we are assuming that the line segment joining $a, b$ does not pass through $z=0$. If we set $|w(z)|=\left|w\left(a+r e^{i \theta}\right)\right|=W(r)$, then (as in the proof of Theorem 1.1) $\left|w^{\prime}(z)\right| \geqq\left|W^{\prime}(r)\right|$, and hence

$$
\int_{0}^{R} W^{\prime 2}(r) d r \leqq \int_{0}^{R}\left|p\left(a+r e^{i \theta}\right)\right| W^{2}(r) d r .
$$

Since $W(0)=W(R)=0$, it follows from (3.1) that, whenever

$$
\left|p\left(a+r e^{i \theta}\right)\right| \leqq \frac{1}{4 r^{2}}+\frac{\lambda}{r}-\lambda^{2},
$$

(2) cf. E. Hille, $A$ note on regular singular points, Arkiv för Matematik Astronomi, och Fysik vol. 19A (1925), especially /Theorems 2 and 3. This paper deals with the case that $a$ is real (including the case $a>1 / 4$ ); the technique makes use of an associated integral equation. In addition, using the Green's transform, the author obtains zero-free regions, again in the case that $a$ is real. 
(3.3) is impossible. This provides the basis for the construction of rather extensive zero-free regions. In fact, by (3.2), the inequality (3.4) will be satisfied whenever

$$
\left|a+r e^{i \theta}\right| \geqq r .
$$

If $a \neq 0$, then the equation $\left|a+r e^{i \theta}\right|=r$ is the equation of the perpendicular bisector of the line segment joining $z=0$ and $z=a$. The domain defined by (3.5) is the half-plane bounded by this line which does not contain the point $z=0$. It follows that $w(z)$ has no other zeros in this half-plane, including the boundary. As a matter of fact, the zero-free region of $w(z)$ may be extended to include the interior and circumference of a circle with center at $a$ of radius $|a|$. For, if there were another zero $z=b$ in this region, then on interchanging the roles of $a$ and $b$, we see that $a$ would lie in the zero-free half-plane corresponding to $z=b$.

Using Theorem 1.1 we may enlarge this zero-free region still further. Suppose that $z=b$ is another zero of $w(z)$, where again we assume that the line segment $(a, b)$ does not pass through $z=0$. If $\theta=\arg (b-a)$ and $\phi=\arg a$, then the shortest distance from this line segment to the origin is $|a| \sin (\pi$ $-(\theta-\phi))=|a| \sin (\theta-\phi)$. Writing $\psi=\theta-\phi$, we have for all $z$ on this segment

$$
|p(z)| \leqq \frac{1}{4|a|^{2} \sin ^{2} \psi}+\frac{\lambda}{|a| \sin \psi}-\lambda^{2} \leqq \frac{1}{2|a|^{2} \sin ^{2} \psi} .
$$

By Theorem 1.1 (with $\lambda=1, \mu=0, Q(r) \equiv \pi^{2}|b-a|^{-2}$ ), it follows that

$$
1 / 2|a|^{2} \sin ^{2} \psi \geqq \pi^{2}|b-a|^{-2},
$$

or writing $|b-a|=\rho$, that

$$
\rho \geqq 2^{1 / 2} \pi|a| \sin \psi .
$$

Now, looking on $\rho, \psi$ as polar coordinates (with $z=a$ as origin), the curve

$$
\rho=2^{1 / 2} \pi|a| \sin \psi
$$

is a circle of radius $\left(2^{1 / 2} / 2\right) \pi|a|$. This circle has its centre on the line which passes through the point $z=a$ and which is perpendicular to the line joining $z=0$ and $z=a$. The circle passes through the point $z=a$. The solution $w(z)$ which vanishes at $z=a$ has no other zeros in the interior of this circle. Of course there is another such zero-free circle lying entirely on the other side of the line $\arg z=\phi$.

The circle (3.6) intersects the circle of radius $|a|$ drawn about the point $z=a$ in a point $\zeta$, and intersects the boundary of the half-plane $\left|a+r e^{i \theta}\right| \geqq r$ in a point $\eta$. It may be shown by elementary geometry that the points $z=0$, $z=\zeta, z=\eta$ all lie on a line. If the angle between this line and the line joining $z=0$ to $z=a$ is denoted by $\beta$, then we find $\beta>83^{\circ} 29^{\prime}$. Clearly, then, we have a zero-free sector situated symmetrically about the line joining $z=0$ and $z=a$, 
having $z=0$ as vertex, and an included angle of at least $166^{\circ} 58^{\prime}$.

So far, we have excluded the case $a=0$. We assert that in case $w(z)$ is analytic at $z=0$, or more generally, if $w^{\prime}(z)$ remains bounded near $z=0$, then if $w(0)=0, w(z)$ has no other zeros in $D$. This will follow from (3.5) provided (3.3) holds in this case. However, since $\left|W^{\prime}(r)\right| \leqq\left|w^{\prime}(z)\right|, W^{\prime}(r)$ is bounded near $r=0$, and one sees that Lemma 1.1 holds for such functions $y(r)=W(r)$, so that (3.3) remains valid.

We collect our results as

Theorem 3.1. Let $D$ be any convex domain of the complex plane included in a sector of angle $83^{\circ} 29^{\prime}$ with vertex at $z=0$. Suppose $p(z)$ is analytic throughout $D$ except possibly at $z=0$ (if $0 \in D$ ), and that

$$
|p(z)| \leqq \frac{1}{4|z|^{2}}+\frac{\lambda}{|z|}-\lambda^{2},
$$

for all $z \in D$. Then, except for a possible zero at $z=0$, no solution of

$$
w^{\prime \prime}+p(z) w=0
$$

has more than one zero in D. Moreover, if a solution $w(z)$ of $(1.2)$ has $w(0)=0$, then $w(z)$ has no other zeros in $D$ provided $w^{\prime}(z)$ remains bounded near $z=0$.

CoROLlaRY 3.2. If $p(z)$ is analytic in any convex region $D$ containing the point $z=0$, except possibly at $z=0$, and satisfies there the condition (3.2), then no branch of any solution of equation (1.2) can have more than 5 zeros in $D$ other than $z=0$. Moreover, any solution w(z) of (1.2) which has w'(z) bounded near $z=0$ and which vanishes at $z=0$ has no other zeros in $D$.

In general, we are not able to reduce the number 5 to 4 , since a branch $w(z)$ may have two zeros lying on opposite sides of a branch cut joining $z=0$ to $z=\infty$. If, however, $|w(z)|$ is the same for all branches of a solution, then no branch can have more than 4 zeros other than $z=0$. The same remark clearly holds for solutions of the form $z^{\alpha} v(z)$, where $v(z)$ is analytic at $z=0$. Since $z=0$ is a regular singular point, equation (1.2) always possesses one such solution.

If $\lambda=0, D$ may be the entire complex plane. In this case, our result is that no solution of $w^{\prime \prime}+p(z) w=0$ has more than 5 zeros in the slit plane. In particular, the equation is nonoscillatory both for large $|z|$ and small $|z|$. This is a generalization of the corresponding result for real differential equations, where the equation $y^{\prime \prime}+a x^{-2} y=0$ plays a critical role. (See Hille [5].)

An example of a class of equations to which the preceding theorem applies is given by Whittaker's equation [16, p. 337],

$$
W^{\prime \prime}+\left\{\frac{1 / 4-m^{2}}{z^{2}}+\frac{\dot{k}}{z}-\frac{1}{4}\right\} W=0,
$$


satisfied by the Whittaker functions $W_{k, m}(z)$. If $0<m^{2}<1 / 2$, then

$$
|p(z)| \leqq \frac{1}{4|z|^{2}}+\frac{|k|}{|z|}-|k|^{2}
$$

for all $z$ in a sufficiently small neighborhood of $z=0$. Hence, for $0<m^{2}<1 / 2$, we may assert that $W_{k, m}(z)$ is nonoscillatory in a neighborhood of $z=0$.

4. A lower bound for the distance between zeros. If $p(z)$ is analytic in a simply-connected region $D$, then every solution $w(z)$ of the equation

$$
w^{\prime \prime}+p(z) w=0
$$

is analytic in $D$ and hence, in particular, no (interior) point of $D$ can be a limit point of zeros of $w(z)$. Normally if $p(z)$ has singularities on the boundary of $D$ the lower bound for the distance between any pair of zeros of any solution of (1.2) is zero. B. Schwarz [12] considered this problem and found criteria insuring a positive lower bound. The following theorem is of the same nature.

THEOREM 4.1. Let $p(z)$ be analytic in the circle $|z|<R$, and set

$$
M(r)=\max _{|z|=r}|p(z)|, \quad 0 \leqq r<R .
$$

Suppose there exists $x_{0}, 0 \leqq x_{0}<R$, such that for each $x, x_{0} \leqq x<R$, the differential equation

$$
\frac{d^{2} y}{d t^{2}}+M\left[\left(t^{2}+x^{2}\right)^{1 / 2}\right] y=0
$$

has a solution $y_{x}(t)$ which does not vanish on the interval $-\left(R^{2}-x^{2}\right)^{1 / 2}<t$ $<\left(R^{2}-x^{2}\right)^{1 / 2}$. Then a lower bound for the distance between any pair of zeros (in $|z|<R)$ of any solution of

$$
w^{\prime \prime}+p(z) w=0
$$

is given by $d=2\left(R^{2}-x_{0}^{2}\right)^{1 / 2}$.

Proof. Assume a solution $w(z)$ of (1.2) has zeros at $z=a, z=b$, where $|b| \leqq|a|<R$, and $0<|b-a|<d$. Let $b^{\prime}$ be the point on the circle $|z|=|a|$ lying on the same side of the line through $z=0$ and $z=a$ as $b$, and such that $\left|b^{\prime}-a\right|=|b-a|$. By a rotation $\zeta=\alpha z,|\alpha|=1$, the points $a, b^{\prime}$ can be brought into a position in the upper half-plane symmetric with respect to the imaginary axis. As in the proof of Theorem 2.1, we may assume that $a, b^{\prime}$ are already in this position.

From the Green's transform we have

$$
\int_{a}^{b}\left|w^{\prime}(z)\right|^{2} d \bar{z}=\int_{a}^{b} p(z)|w(z)|^{2} d z .
$$


Let $z=((a+b) / 2)+t e^{i \theta},|b-a|=2 \eta,|w(z)|=y(t)$. Then

$$
\int_{-\eta}^{\eta} y^{\prime 2}(t) d t \leqq \int_{-\eta}^{\eta} y^{2}(t)\left|p\left(\frac{a+b}{2}+t e^{i \theta}\right)\right| d t .
$$

Since

$$
\begin{aligned}
\left|p\left(\frac{a+b}{2}+t e^{i \theta}\right)\right| & \leqq M\left(\left|\frac{a+b}{2}+t e^{i \theta}\right|\right) \leqq M\left(\left|t+\frac{a+b^{\prime}}{2}\right|\right) \\
& \leqq M\left[\left(t^{2}+|a|^{2}-\eta^{2}\right)^{1 / 2}\right],
\end{aligned}
$$

the last inequality reduces to

$$
\int_{-\eta}^{\eta} y^{\prime 2}(t) d t \leqq \int_{-\eta}^{\eta} y^{2}(t) M\left[\left(t^{2}+|a|^{2}-\eta^{2}\right)^{1 / 2}\right] d t .
$$

Suppose first that $|a|^{2}-\eta^{2}>x_{0}^{2}$. Then the equation (4.1), with $x^{2}=|a|^{2}$ $-\eta^{2}$, has a solution which does not vanish on the interval

$$
-\left(R^{2}-|a|^{2}+\eta^{2}\right)^{1 / 2}<t<\left(R^{2}-|a|^{2}+\eta^{2}\right)^{1 / 2},
$$

hence not on the closed interval $-\eta \leqq t \leqq \eta$. By the remark following Lemma 1.1 , we then have

$$
\int_{-\eta}^{\eta} y^{\prime 2}(t) d t>\int_{-\eta}^{\eta} y^{2}(t) M\left[\left(t^{2}+|a|^{2}-\eta^{2}\right)^{1 / 2}\right] d t
$$

but this contradicts (4.2), proving the theorem in this case.

Finally, if $|a|^{2}-\eta^{2} \leqq x_{0}^{2}$, then (4.2) may be replaced by the inequality

$$
\int_{-\eta}^{\eta} y^{\prime 2}(t) d t \leqq \int_{-\eta}^{\eta} y^{2}(t) M\left[\left(t^{2}+x_{0}^{2}\right)^{1 / 2}\right] d t,
$$

and the proof follows as before, on noting that $\eta<\left(R^{2}-x_{0}^{2}\right)^{1 / 2}$.

We note that if the hypotheses are satisfied for $x_{0}=0$, then the thorem asserts that $w^{\prime \prime}+p(z) w=0$ is disconjugate in $|z|<R$. The theorem includes several sharp cases, as noted in examples (i), (ii) below.

$$
|p(z)| \leqq 2 /\left(R^{2}-|z|^{2}\right), \quad 0 \leqq|z|<R .
$$

In this case the differential equation

$$
\frac{d^{2} y}{d t^{2}}+\frac{2}{\left(R^{2}-x^{2}\right)-t^{2}} y=0
$$

has the solution $y_{x}(t)=\left(R^{2}-x^{2}\right)-t^{2}$ satisfying the hypotheses of the theorem with $x_{0}=0$. The constant 2 is sharp for disconjugacy. As noted following Corollary 2.1, this result was first announced by Pokornyi [11], and is also included in a theorem of Nehari [9]. 


$$
|p(z)| \leqq \pi^{2} / 4 R^{2}
$$$$
0 \leqq|z|<R \text {. }
$$

The differential equation

$$
d^{2} y / d t^{2}+\left(\pi^{2} / 4 R^{2}\right) y=0
$$

has the solution $y(t)=\cos (\pi t / 2 R)$ satisfying the hypotheses of the theorem with $x_{0}=0$. This is just Corollary 1.1 ; it is also sharp for disconjugacy.

In general, equation (4.1) cannot be solved explicitly for each value of the parameter $x$. In such cases the following corollary will of ten be useful.

Corollary 4.1. The hypotheses of Theorem 2.1 will be satisfied if the differential equation

$$
d^{2} y / d r^{2}+M(r) y=0
$$

has a solution $y(r)$ which does not vanish on the interval $-R<r<R$, and provided that for each $x, x_{0} \leqq x<R$, we have

$$
M\left[\left(t^{2}+x^{2}\right)^{1 / 2}\right] \leqq \frac{R^{2}}{R^{2}-x^{2}} M\left(\frac{R t}{\left(R^{2}-x^{2}\right)^{1 / 2}}\right)
$$

for $|t|<\left(R^{2}-x^{2}\right)^{1 / 2}$.

In order to prove this we note that the substitutions $r=R t\left(R^{2}-x^{2}\right)^{-1 / 2}$, $y(r)=Y(t)$ transform equation (4.4) into the equation

$$
\frac{d^{2} Y}{d t^{2}}+\frac{R^{2}}{R^{2}-x^{2}} M\left(\frac{R t}{\left(R^{2}-x^{2}\right)^{1 / 2}}\right) Y=0 .
$$

For each $x, 0 \leqq x<R$, this equation has a solution $Y(t)$ which does not vanish on the interval $-\left(R^{2}-x^{2}\right)^{1 / 2}<t<\left(R^{2}-x^{2}\right)^{1 / 2}$. The result now follows from (4.5) and the Sturm comparison theorem.

In the case $x_{0}=0$, Pokornyi [11], using a somewhat different notation, announced (without proof) that (4.5) was sufficient for disconjugacy. That (4.5) alone is not sufficient is easily seen by considering the case where $p(z) \equiv C, C>\pi^{2} / 4 R^{2}$.

It may also be of interest to note that in the case of disconjugacy, i.e., $x_{0}=0$, the condition (4.5) is equivalent to the much simpler condition that the function $\left(R^{2}-r^{2}\right) M(r)$ be nonincreasing for $0 \leqq r<R$, so that in this case the result is contained in Corollary 2.1. To see this, we first note that (4.5) is equivalent to the condition

$$
\frac{R^{2}-x^{2}}{R^{2}} M\left(\frac{1}{R}\left[R^{2} x^{2}+\left(R^{2}-x^{2}\right) r^{2}\right]^{1 / 2}\right) \leqq M(r), \quad-R<r<R .
$$

This is just (4.5) with $t=R^{-1} r\left(R^{2}-x^{2}\right)^{1 / 2}$. The result now follows precisely as in the case of (2.3) and (2.4).

As an example for Corollary 4.1, suppose that 


$$
M(r) \leqq Q(r)=\nu(\nu+1) /\left(1-r^{2}\right),
$$

where $\nu$ is an odd, positive integer. The differential equation $y^{\prime \prime}+Q(r) y=0$ has the solution $y=\left(1-r^{2}\right) P_{\nu}^{\prime}(r)$, where $P_{\nu}$ is the Legendre polynomial of degree $\nu$. If $\beta_{0}$ is the least positive zero of $P_{\nu}^{\prime}(r)$, then one easily verifies that the corollary holds with $R=\beta_{0}, x_{0}=0$. We thus obtain a sharp radius of disconjugacy $|z|=\beta_{0}$, i.e., $w^{\prime \prime}+p(z) w=0$ is disconjugate in $|z|<\beta_{0}$, if $M(r)$ satisfies $(4.7)$, and the constant $\nu(\nu+1)$ cannot be increased.

At the beginning of this section we mentioned the fact that B. Schwarz obtained the first criteria insuring the existence of a positive lower bound for the distance between any pair of zeros of any solution of (1.2). One of these criteria may be stated as follows: If $p(z)$ is analytic in $|z|<1$, and if

$$
\max _{|z|=r}|p(z)|=M(r) \leqq \frac{1}{1-r^{2}}, \quad x_{0} \leqq r<1,
$$

where $0 \leqq x_{0}<1$, then a lower bound for the distance between any pair of zeros (in $|z|<1$ ) of any solution of $(1.2)$ is given by $2\left(1-x_{0}^{2}\right)^{1 / 2}$.

Using Theorem 4.1, we may improve the constant 1 in (4.8) to 2 . In fact, if

$$
M(r) \leqq 2 /\left(1-r^{2}\right),
$$

$$
x_{0} \leqq r<1 \text {, }
$$

then

$$
M\left[\left(t^{2}+x^{2}\right)^{1 / 2}\right] \leqq \frac{2}{1-x^{2}-t^{2}}, \quad|t|<\left(1-x^{2}\right)^{1 / 2},
$$

for each $x, x_{0} \leqq x<1$. Since, as noted in example (i), the differential equation

$$
\frac{d^{2} y}{d t^{2}}+\frac{2}{1-x^{2}-t^{2}} y=0
$$

has a solution $y_{x}(t)$ which does not vanish on the interval $-\left(1-x^{2}\right)^{1 / 2}<t$ $<\left(1-x^{2}\right)^{1 / 2}$, the result now follows from Theorem 4.1, using the inequality (4.9) and the Sturm comparison theorem. The constant 2 cannot be improved since the theorem is sharp in the case of disconjugacy $\left(x_{0}=0\right)$.

5. Some results on real differential equations. The lemmas of this section are used in the sequel, but are not without interest for their own sake.

LEMma 5.1. Let the functions $q(x), q_{1}(x)$ be continuous, non-negative, and symmetric on the interval $-\alpha<x<\alpha$. Suppose the differential equation

$$
y^{\prime \prime}+q(x) y=0
$$

has a solution $y(x)$ which is symmetric with respect to $x=0$, and is positive on the interval $-\alpha<x<\alpha$. If

$$
\int_{0}^{x} q_{1}(t) y(t) d t \leqq \int_{0}^{x} q(t) y(t) d t, \quad 0<x<\alpha,
$$


then the differential equation

$$
y_{1}^{\prime \prime}+q_{1}(x) y_{1}=0
$$

has a solution which does not vanish on the interval $-\alpha<x<\alpha$.

Proof. Let $y_{1}(x)$ be a symmetric solution of (5.3). (Such a solution obviously exists.) Assume $y_{1}(\beta)=y_{1}(-\beta)=0$, where $0<\beta<\alpha$. We may assume that $y_{1}(0)>0$. Then we have $y_{1}(x)>0,-\beta<x<\beta$, and $y_{1}^{\prime}(x) \geqq 0,-\beta \leqq x \leqq 0$. Similarly, $y(x)>0,-\alpha<x<\alpha$, and $y^{\prime}(x) \geqq 0,-\alpha \leqq x \leqq 0$.

Now, multiplying (5.1) by $y_{1}(x),(5.3)$ by $y(x)$, subtracting and integrating between the limits $x=-\beta$ to $x=0$, we have

$$
y_{1}(x) y^{\prime}(x)-\left.y(x) y_{1}^{\prime}(x)\right|_{-\beta} ^{0}=\int_{-\beta}^{0}\left[q_{1}(t)-q(t)\right] y(t) y_{1}(t) d t .
$$

Since $y^{\prime}(0)=y_{1}^{\prime}(0)=y_{1}(-\beta)=0$, this reduces to

$$
\begin{aligned}
y(-\beta) y_{1}^{\prime}(-\beta) & =\int_{-\beta}^{0}\left[q_{1}(t)-q(t)\right] y(t) y_{1}(t) d t \\
& =y_{1}(0) \int_{-x}^{0}\left[q_{1}(t)-q(t)\right] y(t) d t, \quad 0<x<\beta,
\end{aligned}
$$

where we have used the second integral mean value theorem. Now $y(-\beta) y_{1}^{\prime}$ $(-\beta)>0$, while the right side of the last equation is clearly not positive by (5.2). Hence we have a contradiction, and it follows that the symmetric solution of (5.3) has no zeros on $-\alpha<x<\alpha$.

We now apply Lemma 5.1 to prove an interesting result which was announced (without proof) by V. V. Pokornyi [11].

LеммA 5.2. Let $q(x)$ be continuous and non-negative on the interval $-\alpha \leqq x$ $\leqq \alpha$. Moreover, suppose that. $q(x)$ is symmetric with respect to $x=0$, and nonincreasing for $0 \leqq x \leqq \alpha$. Suppose that the differential equation (5.1) has a solution which does not vanish on the interval $-\alpha<x<\alpha$. Set

$$
q_{1}(x)=\left\{\begin{array}{lr}
q(\alpha-x), & 0 \leqq x \leqq \alpha, \\
q_{1}(-x), & -\alpha \leqq x \leqq 0 .
\end{array}\right.
$$

Then the equation (5.3) has a solution with the same property.

Proof. Let $y(x)$ be the symmetric solution of equation (5.1) with $y(0)>0$. Then $y(x)>0,-\alpha<x<\alpha$. If we set

$$
I_{x}=\int_{0}^{x}\left[q_{1}(t)-q(t)\right] y(t) d t, \quad 0<x \leqq \alpha
$$

then $I_{x} \leqq I_{\alpha}$ clearly. Now 


$$
\begin{aligned}
I_{\alpha} & =\int_{0}^{\alpha}[q(\alpha-t)-q(t)] y(t) d t \\
& =\int_{0}^{\alpha / 2}[q(\alpha-t)-q(t)][y(t)-y(\alpha-t)] d t .
\end{aligned}
$$

For $0 \leqq t \leqq \alpha / 2$, we have $q(\alpha-t) \leqq q(t)$ by hypothesis. Moreover, since $y^{\prime}(t)<0$ for $0<t \leqq \alpha$, we also have $y(t)>y(\alpha-t)$. Hence $I_{\alpha} \leqq 0$, and the result follows from Lemma 5.1.

LemMA 5.3. Let $q(x)$ be continuous and non-negative on the interval $-\alpha \leqq x$ $\leqq \alpha$, and symmetric with respect to $x=0$, so that $q(-x)=q(x)$. Suppose the differential equation

$$
y^{\prime \prime}+q(x) y=0
$$

has a solution which does not vanish on the interval $-\alpha<x<\alpha$. Suppose $0<\beta<\alpha$, and set

$$
q_{1}(x)= \begin{cases}q(x+\beta), & 0 \leqq x \leqq \alpha-\beta, \\ q_{1}(-x), & \beta-\alpha \leqq x \leqq 0 .\end{cases}
$$

Then the differential equation

$$
y^{\prime \prime}+q_{1}(x) y=0
$$

has a solution which does not vanish on the interval $\beta-\alpha<x<\alpha-\beta$.

Proof. Since the functions $q(x), q_{1}(x)$ are symmetric about $x=0$, there exist solutions $y(x), y_{1}(x)$ of equations (5.4), (5.5) respectively which are symmetric about $x=0$. Hence $y^{\prime}(0)=y_{1}^{\prime}(0)=0$. Without loss of generality we may assume that $y(0)=y_{1}(0)>0$. Moreover, by hypothesis we must then have $y(-\alpha)=y(\alpha) \geqq 0, y(x)>0,-\alpha<x<\alpha$.

Now suppose the conclusion does not hold. Then there exists $\gamma<\alpha-\beta$ such that $y_{1}(-\gamma)=y_{1}(\gamma)=0, y_{1}(x)>0,-\gamma<x<\gamma$. On the interval $\beta-\alpha \leqq x$ $\leqq 0$, the function $y_{2}(x)=y(-x+\beta)$ is a solution of (5.5). Hence, by Abel's identity, we have $y_{2} y_{1}^{\prime}-y_{1} y_{2}^{\prime} \equiv$ constant, i.e.,

$$
y(-x+\beta) y_{1}^{\prime}(x)+y_{1}(x) y^{\prime}(-x+\beta) \equiv k, \quad \beta-\alpha \leqq x \leqq 0 .
$$

Evaluating this expression for $x=-\gamma$, and $x=0$, we obtain $y(\gamma+\beta) y_{1}^{\prime}(-\gamma)$ $=k$ and $y_{1}(0) y^{\prime}(\beta)=k$. But this is impossible since $y(\gamma+\beta) y_{1}^{\prime}(-\gamma)>0$, while $y_{1}(0) y^{\prime}(\beta) \leqq 0$.

LEMмA 5.4. Let $q(x)$ be continuous for $-\alpha<x<\alpha$, with $q(-x)=q(x) \geqq 0$, and suppose that $q(x)$ is nondecreasing for $0 \leqq x<\alpha$. Consider the differential equation

$$
y^{\prime \prime}+q(x) y=0
$$


(A) If equation (5.6) has a solution $y(x)$ with $y(0)=0$ and $y^{\prime}(x)>0$, $0 \leqq x<\alpha$, then (5.6) is disconiugate on $-\alpha<x<\alpha$.

(B) If (5.6) is disconjugate on $0<x<\alpha$, and $y(x)$ is a solution of (5.6) with $y(0)=0, y^{\prime}(0)>0$, then $y^{\prime}(x)>0,0 \leqq x<\alpha / 2$.

Proof. For arbitrary $\alpha_{1}<\alpha$ define a function

$$
Q(x)=\left\{\begin{array}{lr}
q\left(\alpha_{1}-x\right), & 0 \leqq x \leqq \alpha_{1}, \\
q\left(\alpha_{1}+x\right), & -\alpha_{1} \leqq x \leqq 0 .
\end{array}\right.
$$

Let $y_{1}(x)$ be a solution of (5.6) with $y_{1}^{\prime}\left(\alpha_{1}\right)=0, y_{1}\left(\alpha_{1}\right)>0$. If we define $Y(x)$ $=y_{1}\left(\alpha_{1}+x\right),-\alpha_{1} \leqq x \leqq 0, Y(x) \equiv Y(-x)$, then $Y(x)$ is a solution of the differential equation $Y^{\prime \prime}+Q(x) Y=0$. Moreover, $Y(x)>0$ for $-\alpha_{1} \leqq x \leqq \alpha_{1}$; this follows from Abel's identity

$$
y(x) y_{1}^{\prime}(x)-y_{1}(x) y^{\prime}(x) \equiv k, \quad 0 \leqq x \leqq \alpha_{1} .
$$

For, at $x=\alpha_{1}$, we find $k<0$. If we assume that $Y(a)=0,0 \leqq a \leqq \alpha_{1}$, then $Y(-a)$ $=y_{1}\left(\alpha_{1}-a\right)=0$, and we obtain $k=y\left(\alpha_{1}-a\right) y_{1}^{\prime}\left(\alpha_{1}-a\right)>0$.

Assertion (A) now follows easily from Lemma 5.2. To see this, let

$$
O_{1}(x)=\left\{\begin{array}{lr}
Q\left(\alpha_{1}-x\right), & 0 \leqq x \leqq \alpha_{1}, \\
Q_{1}(-x), & -\alpha_{1} \leqq x \leqq 0 .
\end{array}\right.
$$

Then the differential equation $y^{\prime \prime}+Q_{1}(x) y=0$ has a solution which does not vanish on the interval $-\alpha_{1}<x<\alpha_{1}$. Since $Q_{1}(x) \equiv q(x)$, and $\alpha_{1}<\alpha$ is arbitrary, assertion (A) is proved.

To prove assertion (B), assume that $y^{\prime}\left(x_{1}\right)=0,0<x_{1}<\alpha / 2$. Set

$$
Q(x)=\left\{\begin{array}{lr}
q(x), & 0 \leqq x \leqq x_{1}, \\
q\left(2 x_{1}-x\right), & x_{1} \leqq x \leqq 2 x_{1} .
\end{array}\right.
$$

Then the function

$$
Y(x)=\left\{\begin{array}{lr}
y(x), & 0 \leqq x \leqq x_{1}, \\
y\left(2 x_{1}-x\right), & x_{1} \leqq x \leqq 2 x_{1},
\end{array}\right.
$$

is a solution of the differential equation $Y^{\prime \prime}+Q(x) Y=0$ which vanishes at $x=0, x=2 x_{1}<\alpha$. But this is impossible by the Sturm comparison theorem since $Q(x) \leqq q(x), 0 \leqq x \leqq 2 x_{1}$.

6. On some results of V. V. Pokornyi. Suppose that $p(z)$ is analytic in a circular region $|z|<R$. In a paper published in 1951, Pokornyi [11], announced (without proof) two criteria which assure the disconjugacy of the equation $w^{\prime \prime}+p(z) w=0$ in smaller circular regions. Both of these results are contained in the following theorem, which is itself a corollary of Theorem 4.1.

Theorem 6.1. Let $p(z)$ be analytic in $|z|<R$. Set 


$$
M(r)=\max _{|z|=r}|p(z)|, \quad 0 \leqq r<R .
$$

If, for each $x, 0 \leqq x<R$, the differential equation

$$
d^{2} y / d t^{2}+M\left[\left(t^{2}+x^{2}\right)^{1 / 2}\right] y=0
$$

has a solution $y_{x}(t)$ such that $y_{x}(0)=0$ and $y_{x}^{\prime}(t)>0,0 \leqq t<\left(R^{2}-x^{2}\right)^{1 / 2}$, then the differential equation

$$
w^{\prime \prime}+p(z) w=0
$$

is disconjugate in $|z|<R$.

Proof. By Lemma 5.4 (A), it follows that for each $x, 0 \leqq x<R$, equation (6.1) is disconjugate on the interval $-\left(R^{2}-x^{2}\right)^{1 / 2}<t<\left(R^{2}-x^{2}\right)^{1 / 2}$. The result now follows from Theorem 4.1, with $x_{0}=0$.

Corallary 6.1 (Pokornyi). If the differential equation

$$
y^{\prime \prime}+M(t) y=0
$$

has a solution $y(t)$ with $y(0)=0$, and if $y^{\prime}\left(R_{0}\right)=0$, where $R_{0}$ is the least positive zero of $y^{\prime}(t)$, then equation (1.2) is disconjugate in $|z|<R_{0} / 2^{1 / 2}$.

To prove this result we define, for each $x, 0 \leqq x<R_{0} / 2^{1 / 2}$, the function $M_{x}(t)=M(t+x), 0 \leqq t \leqq\left[\left(R_{0}^{2} / 2\right)-x^{2}\right]^{1 / 2}$. We note that $M_{x}(t)$ is well-defined since $t+x<R_{0}$. Let $y_{x}(t)$ be a solution of the differential equation

$$
y_{x}^{\prime \prime}+M_{x}(t) y_{x}=0
$$

such that $y_{x}(0)=0, y_{x}^{\prime}(0)>0$. Then $y_{x}^{\prime}(t)>0,0 \leqq t \leqq\left[\left(R_{0}^{2} / 2\right)-x^{2}\right]^{1 / 2}$, as may be seen from Abel's identity applied to the solutions $y(x+t), y_{x}(t)$ of $(6.3)$.

Now, if $Y_{x}(t)$ is a solution of equation (6.1) such that $Y_{x}(0)=0, Y_{x}^{\prime}(0)>0$, then $Y_{x}^{\prime}(t)>0,0 \leqq t \leqq\left[\left(R_{0}^{2} / 2\right)-x^{2}\right]^{1 / 2}$. This follows from the fact that $Y_{x}(t)>0$ on this interval (as is readily seen by applying the Sturm comparison theorem to equations (6.1) and (6.3)), together with the identity

$$
y_{x}(t) Y_{x}^{\prime}(t)-Y_{x}(t) y_{x}^{\prime}(t) \equiv \int_{0}^{t} y_{x}(u) Y_{x}(u)\left[M_{x}(u)-M\left[\left(u^{2}+x^{2}\right)^{1 / 2}\right]\right] d u .
$$

The result now follows from Theorem 6.1, with $R$ replaced by $R_{0} / 2^{1 / 2}$. let

Corollary 6.2 (Pokornyi). Let $p(z)$ be analytic in the circle $|z|<2 R$, and

$$
\max _{|z|=r}|p(z)|=M(r), \quad 0 \leqq r<2 R .
$$

If the differential equation

$$
y^{\prime \prime}+M(r) y=0
$$


has a solution which does not vanish on the interval $0<r<2 R$, then equation (1.2) is disconjugate in $|z|<2 R / 5^{1 / 2}$.

The proof is similar to that of Corollary 6.1. For each $x, 0 \leqq x<2 R / 5^{1 / 2}$, one sees that the differential equation $y^{\prime \prime}+M(t+x) y=0$ has a solution which does not vanish on the interval $0 \leqq t \leqq 2\left[\left(4 R^{2} / 5\right)-x^{2}\right]^{1 / 2}$. Since $\left(t^{2}+x^{2}\right)^{1 / 2}$ $\leqq t+x$, equation (6.1) has a solution with the same property. The corollary now follows from Lemma 5.4 (B), and Theorem 6.1.

It may be of interest to note that there is a "two-sided" criterion corresponding to Corollary 6.1. This criterion is again a corollary of Theorem 4.1, and it contains Corollary 6.1. We may state it as

Lemma $6.1\left(^{3}\right)$. Let $p(z)$ be analytic in $|z|<R$, and let $M(r)$ be defined as before. If the differential equation (6.2) has a solution which does not vanish on the interval $-R<r<R$, then equation (1.2) is disconjugate in $|z|<R / 2^{1 / 2}$.

Proof. As in Corollary 6.1, for each $x, 0 \leqq x<R / 2^{1 / 2}$, we define the function $M_{x}(t)=M(t+x), 0 \leqq t \leqq\left[\left(R^{2} / 2\right)-x^{2}\right]^{1 / 2}$, and by symmetry for $t<0$. By Lemma 5.3 (with $\beta=x, \alpha=x+\left[\left(R^{2} / 2\right)-x^{2}\right]^{1 / 2}<R$ ) it follows that the differential equation $y^{\prime \prime}+M_{x}(t) y=0$ has a solution which does not vanish on the interval $-\left[\left(R^{2} / 2\right)-x^{2}\right]^{1 / 2}<t<\left[\left(R^{2} / 2\right)-x^{2}\right]^{1 / 2}$. Since $\left(t^{2}+x^{2}\right)^{1 / 2} \leqq t+x$, the differential equation

$$
\frac{d^{2} y}{d t^{2}}+M\left[\left(t^{2}+x^{2}\right)^{1 / 2}\right] y=0
$$

has a solution with the same property. The lemma now follows from Theorem 4.1 (with $R$ replaced by $R / 2^{1 / 2}$, and $x_{0}=0$ ).

Lemma 5.4 (A) shows that this two-sided criterion is stronger than the corresponding one-sided criterion of Corollary 6.1.

7. The Green's function. Let $p(z)$ be analytic in a simply-connected region $D$, and suppose that $w(z)$ is a solution of the equation

$$
w^{\prime \prime}+p(z) w=0 .
$$

If $w(a)=w(b)=0$, where $a \in D, b \in D$, then as pointed out in [8], the wellknown identity

$$
w(\zeta)=\int_{a}^{b} g(z, \zeta) p(z) w(z) d z
$$

is still valid if the functions involved are analytic, and the integration path is any path in $D$ joining $a$ and $b$, rather than a linear segment. Here, $g(z, \zeta)$ is the Green's function of the differential system $w^{\prime \prime}=0, w(a)=w(b)=0$. That is, if $\zeta$ denotes an arbitrary (interior) point of the path $C$ joining $a$ and $b$, and

(3) This theorem was proved independently by Binyamin Schwarz, but was not published. 
if $C_{1}, C_{2}$ denote the sub-arcs of $C$ which join $a$ and $\zeta$, and $\zeta$ and $b$, respectively, then

$$
g(z, \zeta)= \begin{cases}\frac{(z-a)(b-\zeta)}{b-a}, & z \in C_{1}, \\ \frac{(\zeta-a)(b-z)}{b-a}, & z \in C_{2} .\end{cases}
$$

With Nehari, we choose $\zeta$ to be the point (or one of the points) of $C$ at which $|w(\zeta)|$ assumes its maximum value on $C$. From (7.1) we then obtain

$$
1 \leqq \int_{a}^{b}|g(z, \zeta)| \cdot|p(z)| \cdot|d z|
$$

We now take $C$ to be the straight line path joining $a$ and $b$. If we set $z=a+r e^{i \theta \text {, }}$ $\zeta=a+\rho e^{i \theta}, b=a+R e^{i \theta}$, then

$$
|g(z, \zeta)|= \begin{cases}r(R-\rho) / R, & 0 \leqq r \leqq \rho, \\ \rho(R-r) / R, & \rho \leqq r \leqq R .\end{cases}
$$

Notice that, in all cases,

$$
|g(\dot{z}, \zeta)| \leqq r(R-r) / R
$$

Hence, by (7.3),

$$
R \leqq \int_{0}^{R} r(R-r)\left|p\left(a+r e^{i \theta}\right)\right| d r .
$$

For later use we are interested in the direct estimate (7.3), which may be rewritten in the form

$$
R \leqq \int_{0}^{\rho} r(R-\rho)\left|p\left(a+r e^{i \theta}\right)\right| d r+\int_{\rho}^{R} \rho(R-r)\left|p\left(a+r e^{i \theta}\right)\right| d r .
$$

Denoting the right side of this inequality by $f(\rho)$, one finds that $f(\rho)$ has an absolute maximum which occurs when

$$
\int_{0}^{\rho} r\left|p\left(a+r e^{i \theta}\right)\right| d r=\int_{\rho}^{R}(R-r)\left|p\left(a+r e^{i \theta}\right)\right| d r
$$

and that

$$
R \leqq R \int_{0}^{p} r\left|p\left(a+r e^{i \theta}\right)\right| d r=R \int_{0}^{R}(R-r)\left|p\left(a+r e^{i \theta}\right)\right| d r .
$$

Thus we have 


$$
1 \leqq\left\{\begin{array}{l}
\int_{0}^{p} r\left|p\left(a+r e^{i \theta}\right)\right| d r, \\
\int_{\rho}^{R}(R-r)\left|p\left(a+r e^{i \theta}\right)\right| d r,
\end{array}\right.
$$

where $\rho$ satisfies equation (7.5).

We illustrate the use of (7.4), (7.6) by finding circular zero-free regions of the solution $w(z)$ of the equation

$$
w^{\prime \prime}+z^{n} w=0,
$$

where $w(0)=0$. From (7.4) we obtain

$$
1 \leqq R^{n+2} /(n+2)(n+3),
$$

while (7.6) gives

$$
1 \leqq R^{n+2} /\left\{(n+2)^{(2 n+3) /(n+1)}\right\} .
$$

In this case, (7.6) gives the better estimate for $R$, and we conclude that $w(z)$ has no other zeros in the circle $|z|<R$, where

$$
R=(n+2)^{(2 n+3) /(n+1)(n+2)} \text {. }
$$

The inequality (7.4), in the real case, is contained in a more general criterion due to Hartman and Wintner [2].

8. Nonoscillation in strips and sectors. The developments of this section are closely related to some of the work in [8].

TheOREM 8.1. Let $p(z)$ be analytic in, and on the boundary lines of, an infinite region $D$ of the half-plane $x \geqq x_{0}$, which includes the real axis for $x \geqq x_{0}$, and is included between the lines $y= \pm M,(z=x+i y)$. Suppose that

$$
\begin{aligned}
& \int_{x_{0}}^{\infty} x|p(x)| d x<\infty, \\
& |p(x+i y)| \leqq \epsilon(x), \quad x \geqq x_{0}, x+i y \in D,
\end{aligned}
$$

where $\epsilon(x) \rightarrow 0$ as $x \rightarrow \infty$. Then the differential equation

$$
w^{\prime \prime}+p(z) w=0
$$

is nonoscillatory in D. Moreover, if $p(z)$ is real and does not change sign for large real $z$, and if a solution $w(z)$ has only a finite number of real zeros, then $w(z)$ has only a finite number of zeros throughout $D$ even if (8.1) is replaced by the weaker condition

$$
\int_{x_{0}}^{\infty}|p(x)| d x<\infty
$$


In particular, if the (real) differential equation

$$
w^{\prime \prime}(x)+p(x) w(x)=0
$$

is nonoscillatory (in the real sense), then (1.2) is nonoscillatory in $D$ whenever (8.2) and (8.3) are satisfied.

Proof. Assume there exists a solution $w(z)$ of (1.2) which has an infinity of zeros $\left\{a_{n}\right\}$ in $D$. We may assume that all $\operatorname{Im}\left\{a_{n}\right\} \geqq 0$, that $\operatorname{Re}\left\{a_{n}\right\}$ $>\max \left(M, x_{0}\right)$, and that (if necessary, by choosing subsequences)

$$
a_{n}^{\prime}-a_{n-1}^{\prime}>1, \quad \epsilon\left(a_{n}^{\prime}\right)<n^{-2} \quad\left(a_{n}=a_{n}^{\prime}+i a_{n}^{\prime \prime}\right) .
$$

Now, let $a=a^{\prime}+i a^{\prime \prime}$ and $b=b^{\prime}+i b^{\prime \prime}$ be a consecutive pair of these zeros. Let $C$ be the path joining $a$ and $b$ consisting of the three straight-line segments $L_{1}, L_{2}, L_{3}$, where $L_{1}$ is the vertical line joining $z=a$ to $z=a^{\prime}, L_{2}$ is the segment of the real axis from $x=a^{\prime}$ to $x=b^{\prime}$, and $L_{3}$ is the vertical line joining $z=b^{\prime}$ to $z=b$. According to (7.3) we have

$$
1 \leqq \int_{a}^{b}|g(z, \zeta)| \cdot|p(z)| \cdot|d z|
$$

We now majorize $|g|$, according to the position of $\zeta$ on $C$, using (7.2).

CASE I. $\zeta \in L_{1}$. We consider the three sub-cases
(i) $z \in L_{1}$,
(ii) $z \in L_{2}$,
(iii) $z \in L_{3}$.

(i) $z \in L_{1}$.

$$
|g(z, \zeta)| \leqq\left\{\begin{array}{l}
\frac{M\left(M^{2}+\left(b^{\prime}-a^{\prime}\right)^{2}\right)^{1 / 2}}{b^{\prime}-a^{\prime}}<M\left(1+M^{2}\right)^{1 / 2}=M_{1}, \\
\frac{M\left(M^{2}+\left(b^{\prime}-a^{\prime}\right)^{2}\right)^{1 / 2}}{b^{\prime}-a^{\prime}}<M_{1}
\end{array}\right.
$$

(ii) $z \in L_{2}$.

$$
|g(z, \zeta)| \leqq \frac{M\left(M^{2}+\left(b^{\prime}-a^{\prime}\right)^{2}\right)^{1 / 2}}{b^{\prime}-a^{\prime}}<M_{1}
$$

(iii) $z \in L_{3}$.

$$
|g(z, \zeta)| \leqq M^{2} /\left(b^{\prime}-a^{\prime}\right)<M^{2}<M_{1} .
$$

Case II. $\zeta \in L_{2}$.

(i) $z \in L_{1}$.

$$
|g(z, \zeta)| \leqq \frac{M\left(M^{2}+{ }^{\prime}\left(b^{\prime}-a^{\prime}\right)^{2}\right)^{1 / 2}}{b^{\prime}-a^{\prime}}<M_{1}
$$


(ii) $z \in L_{2}$.

$$
|g(z, \zeta)| \leqq\left\{\begin{array}{l}
|z-a| \frac{\left(M^{2}+\left(b^{\prime}-a^{\prime}\right)^{2}\right)^{1 / 2}}{b^{\prime}-a^{\prime}}<x\left(1+M^{2}\right)^{1 / 2}=x M_{2}, \\
|z-a| \frac{|b-z|}{|b-a|} \frac{|\zeta-a|}{|z-a|}<|z-a| \frac{\left(M^{2}+\left(b^{\prime}-a^{\prime}\right)^{2}\right)^{1 / 2}}{b^{\prime}-a^{\prime}},
\end{array}\right.
$$

since $|z-a|^{2} \leqq M^{2}+\left(x-a^{\prime}\right)^{2}<x^{2}$ for $M<a^{\prime}<x$.

$$
1<x M_{2},
$$

(iii) $z \in L_{3}$.

$$
|g(z, \zeta)| \leqq \frac{\left(M^{2}+\left(b^{\prime}-a^{\prime}\right)^{2}\right)^{1 / 2}}{b^{\prime}-a^{\prime}} M<M_{1} .
$$

CASE III. $\zeta \in L_{3}$. The situation is symmetric with that in Case I, and we obtain $|g(z, \zeta)|<M_{1}$.

Now, if we set $K=\max \left(M_{1}, M_{2}\right)$, then we have, by (8.4),

$$
\begin{aligned}
1< & K \int_{0}^{a^{\prime \prime} n}\left|p\left(a_{n}^{\prime}+i y\right)\right| d y+K \int_{a_{n}^{\prime}}^{a_{n}^{\prime}+1} x|p(x)| d x \\
& +K \int_{0}^{a^{\prime \prime} n+1}\left|p\left(a_{n+1}^{\prime}+i y\right)\right| d y .
\end{aligned}
$$

Adding the above inequalities from $n=1$ to $n=m$, we obtain

$$
m<2 K \sum_{n=1}^{m+1} \int_{0}^{a^{\prime \prime} n}\left|p\left(a_{n}^{\prime}+i y\right)\right| d y+K \int_{a^{\prime}{ }_{1}}^{a^{\prime} m+1} x|p(x)| d x,
$$

whence

$$
\begin{aligned}
& m<2 K \sum_{n=1}^{m+1} a_{n}^{\prime \prime} \epsilon\left(a_{n}^{\prime}\right)+K \int_{x_{0}}^{\infty} x|p(x)| d x, \\
& m<2 K M \sum_{n=1}^{\infty} n^{-2}+K \int_{x_{0}}^{\infty} x|p(x)| d x .
\end{aligned}
$$

Since the right side of this inequality is independent of $m$, we have a contradiction, and the main part of the theorem is proved.

To prove the last part of the theorem, suppose that $w(z)$ is a solution of (1.2) and that $w(x) \neq 0$ for $x \geqq x_{1}$. On the real axis we have

$$
w^{\prime \prime}(x)=u^{\prime \prime}(x)+i v^{\prime \prime}(x)=-p(x)\{u(x)+i v(x)\}=-p(x) w(x),
$$

so that

$$
u^{\prime \prime}(x)=-p(x) u(x), \quad v^{\prime \prime}(x)=-p(x) v(x) .
$$


Multiplying the first of these equations by $u(x)$, the second by $v(x)$, adding and integrating, we obtain

$$
\begin{aligned}
u(x) u^{\prime}(x)+v(x) v^{\prime}(x)= & u\left(x_{1}\right) u^{\prime}\left(x_{1}\right)+v\left(x_{1}\right) v^{\prime}\left(x_{1}\right) \\
& -\int_{x_{1}}^{x} p(t)\left\{u^{2}(t)+v^{2}(t)\right\} d t .
\end{aligned}
$$

Since $p(t)$ does not change sign for $t \geqq x_{1}$, it follows that the left side is always of the same sign for sufficiently large $x>x_{1}$; the same property then holds for

$$
\frac{d}{d x}|w(x)|=\left\{u(x) u^{\prime}(x)+v(x) v^{\prime}(x)\right\} \cdot|w(x)|^{-1},
$$

so that $|w(x)|$ is monotone for large $x$. By the choice of $\zeta$ in the analysis preceding (7.3), we see that $\zeta \in L_{1}$ or $\zeta \in L_{3}$. The proof then proceeds as before except that $x|p(x)|$ is replaced by $|p(x)|$.

If the real differential equation $w^{\prime \prime}(x)+p(x) w(x)=0$ is nonoscillatory (in the real sense), then no real solution $u(x)$ has more than a finite number of real zeros. Hence the same must be true of any complex solution $w(z)$, with $w(x)=u(x)+i v(x)$. Thus, if the differential equation is nonoscillatory on the real axis, it is nonoscillatory throughout $D$ whenever (8.2) and (8.3) are satisfied, provided $p(x)$ is real and does not change sign for large $x$. In this connection we note that if $p(x)>0$, then Wintner [18] has proved that $\int_{x_{0}}^{\infty} p(x) d x=\infty$ is a sufficient condition for (real) oscillation of the (real) equation $w^{\prime \prime}+p(x) w=0$.

We note that the theorem would be false if (8.1) were relaxed to

$$
\int_{x_{0}}^{\infty} x^{1-\epsilon}|p(x)| d x<\infty, \quad 0<\epsilon<1 .
$$

For, the Euler equation $w^{\prime \prime}+z^{-2} w=0$ has solutions with an infinite number of real zeros $z>1$, while conditions (8.2), (8.6) are satisfied.

On the other hand, if $a \leqq 1 / 4$, then the (real) differential equation $w^{\prime \prime}+a x^{-2} w=0$ is known to be nonoscillatory for $x \geqq 1$. Since conditions (8.2) and (8.3) are satisfied, the (complex) differential equation $w^{\prime \prime}+a z^{-2} w=0$ is nonoscillatory in $D$, whenever $a \leqq 1 / 4$.

Theorem 8.2. Let $D_{r}$ be the closed domain bounded by the lines $x=x_{0}>0$, $y= \pm x^{-r},\left(x_{0} \leqq x<\infty\right)$. Suppose that

$$
\int_{x_{0}}^{\infty} x|p(x)| d x<\infty,
$$

\section{and}

$$
|p(x+i y)| \leqq x^{r} \epsilon(x), \quad x \geqq x_{0}, x+i y \in D_{r}, \text { for } r \geqq 0,
$$


$(8.8)_{2}|p(x+i y)| \leqq x^{2 r} \epsilon(x), \quad x \geqq x_{0}, x+i y \in D_{r},-1<r<0$, where $\epsilon(x) \rightarrow 0$ as $x \rightarrow \infty$. Then the differential equation

$$
w^{\prime \prime}+p(z) w=0
$$

is nonoscillatory in $D_{r}$. Moreover, in the case $r \geqq 0$, if $p(z)$ is real and does not change sign for large real $z$, and a solution $w(z)$ of (1.2) has only a finite number of real zeros, then $w(z)$ has only a finite number of zeros throughout $D_{r}$, even if (8.7) is replaced by the weaker condition

$$
\int_{x_{0}}^{\infty}|p(x)| d x<\infty .
$$

Proof. In the case $r \geqq 0$, the proof of Theorem 8.1 holds (with $M=x_{0}^{-r}$ ) as far as equation (8.5). In this case, however, we now have $a_{n}^{\prime \prime} \leqq\left(a_{n}^{\prime}\right)^{-r}$; using $(8.8)_{1}$ we obtain the same contradiction as before.

For the case $-1<r<0$, the idea of the proof is exactly the same but we require a new estimate of $|g(z, \zeta)|$. In this case we assume that a solution $w(z)$ of (1.2) has an infinite number of zeros $\left\{a_{n}\right\}$ in $D_{r}$ with $\operatorname{Im}\left\{a_{n}\right\} \geqq 0$, $\operatorname{Re}\left\{a_{n}\right\}>1$, and $a_{n}^{\prime}-a_{n-1}^{\prime}>\left(a_{n}^{\prime}\right)^{-r}, \epsilon\left(a_{n}^{\prime}\right)<n^{-2}$. Leaving out the computation, we find that for all positions of $\zeta$,

$$
|g(z, \zeta)| \leqq\left\{\begin{array}{lr}
2^{1 / 2}\left(a^{\prime}\right)^{-r}, & z \in L_{1}, \\
2^{1 / 2} x, & z \in L_{2}, z=x, \\
2^{1 / 2}\left(b^{\prime}\right)^{-r}, & z \in L_{3} .
\end{array}\right.
$$

The proof now proceeds as before, and we obtain

$$
m<2 \cdot 2^{1 / 2} \sum_{n=1}^{\infty} n^{-2}+2^{1 / 2} \int_{x_{0}}^{\infty} x|p(x)| d x .
$$

Again we have a contradiction and the theorem is proved.

The example $w^{\prime \prime}+z^{-2} w=0$ applies also to Theorem 8.2 , showing that the condition (8.7) cannot be relaxed to the condition (8.6).

THEOREM 8.3( $\left(^{4}\right)$. Let $p(z)$ be analytic in, and on the boundary lines of, a sector $S_{\alpha}$ bounded by the lines $y= \pm \alpha x, 0<\alpha<\infty$. Suppose that

$$
\begin{aligned}
& |p(x+i y)| \leqq P(x), \quad x+i y \in S_{\alpha}, \\
& \int_{0}^{\infty} x P(x) d x<\infty .
\end{aligned}
$$

Then the differential equation

(4) Cf. E. Hille [5, Theorem 13]. Cf. also C. T. Taam, Schlicht functions and linear differential equations of second order, Journal of Rational Mechanics and Analysis vol. 4 (1955), especially Theorem 12 . 


$$
w^{\prime \prime}+p(z) w=0
$$

is nonoscillatory in $S_{\alpha}$.

Proof. Assume a solution $w(z)$ of (1.2) has an infinite number of zeros $\left\{a_{n}\right\}$ in $S_{\alpha}$. We assume that $\operatorname{Im}\left\{a_{n}\right\} \geqq 0$, and that $\operatorname{Re}\left\{a_{n}\right\}=a_{n}^{\prime}>n$. Since $S_{\alpha}$ is convex, the line joining $a_{n}, a_{m}$ lies in $S_{\alpha}$. Writing arg $\left(a_{m}-a_{n}\right)=\theta_{n, m}$ and setting $z=a_{n}+r e^{i \theta_{n, m}}$, we have, by (7.6),

$$
1 \leqq \int_{0}^{\rho} r\left|p\left(a_{n}+r e^{i \theta_{n}, m}\right)\right| d r, \quad 0<\rho<\left|a_{m}-a_{n}\right|=R,
$$

hence

$$
1<\int_{0}^{R} r\left|p\left(a_{n}+r e^{i \theta_{n, m}}\right)\right| d r .
$$

If $a_{n}+r e^{i \theta_{n, m}}=x+i y$, then $r=\left(x-a_{n}^{\prime}\right) \sec \theta_{n, m}$. Making this substitution, we have

hence

$$
1<\sec ^{2} \theta_{n, m} \int_{a_{n}^{\prime}}^{a_{m}^{\prime}}\left(x-a_{n}^{\prime}\right)|p(x+i y)| d x
$$

$$
\cos ^{2} \theta_{n, m}<\int_{a_{n}^{\prime}}^{a_{m}^{\prime}}\left(x-a_{n}^{\prime}\right) P(x) d x .
$$

If now we hold $n$ fixed and let $m \rightarrow \infty$, we obtain

$$
\begin{aligned}
\cos ^{2} \gamma & \leqq \cos ^{2}\left(\limsup _{m \rightarrow \infty} \theta_{n, m}\right)=\liminf _{m \rightarrow \infty} \cos ^{2} \theta_{n, m} \leqq \int_{a_{n}^{\prime}}^{\infty}\left(x-a_{n}^{\prime}\right) P(x) d x \\
& <\int_{a_{n}^{\prime}}^{\infty} x P(x) d x<\int_{n}^{\infty} x P(x) d x,
\end{aligned}
$$

where $\alpha=\tan \gamma$. Hence finally

$$
\cos ^{2} \gamma \leqq \lim _{n \rightarrow \infty} \int_{n}^{\infty} x P(x) d x=0 .
$$

This is impossible for $0<\gamma<\pi / 2$, hence the theorem is proved.

As an example, we consider the differential equation

$$
w^{\prime \prime}+e^{-z} w=0 \text {. }
$$

We may take $P(x)=e^{-x}$. Since $\int_{0}^{\infty} x e^{-x} d x<\infty$, it follows that this differential equation is nonoscillatory in any sector $S_{\alpha}, 0<\alpha<\infty$. As a matter of fact, the result obviously holds for any sector

$$
S_{\alpha}^{\prime}: z=x_{0}+r e^{i \phi}, x_{0} \text { real, } 0 \leqq r<\infty, \quad-\pi / 2+\epsilon \leqq \phi \leqq \pi / 2-\epsilon .
$$

However, the above equation admits the explicit solution $w(z)=J_{0}\left(2 e^{-s / 2}\right)$, 
where $J_{0}$ is the Bessel function of order zero. This function has an infinity of zeros on each of the vertical lines $x=x_{n}$, where $2 e^{-x_{n}}=\lambda_{n}$ and $\lambda_{n}$ is the $n$th zero of $J_{0}(x)$. This example shows that the range of the theorem cannot be extended to an entire half-plane, either open or closed.

9. A theorem in the half-plane. None of the results of $\S 8$ applies to a halfplane. The following results apply to a half-plane but are of a somewhat different nature.

LEMмA 9.1. Let $p(z)$ be analytic for $\operatorname{Re}\{z\} \geqq x_{0}$, and set

$$
\max \left\{|p(z)|:\left|z-x_{0}\right|=r, \operatorname{Re}\{z\} \geqq x_{0}\right\}=M(r), \quad 0 \leqq r<\infty .
$$

Let $z=a, z=b$ be two zeros of a solution $w(z)$ of $w^{\prime \prime}+p(z) w=0$ such that $\operatorname{Re}\{a\} \geqq x_{0}, \operatorname{Re}\{b\} \geqq x_{0}$, and $\left|b-x_{0}\right| \geqq\left|a-x_{0}\right|$.

If $M(r)$ is monotone decreasing, and if

$$
\int_{0}^{\left|b-x_{0}\right|} r M(r) d r \leqq 1
$$

then $|b-a|>\left|b-x_{0}\right|$.

Proof. For the proof we may assume $x_{0}=0$. Assume a solution $w(z)$ of $w^{\prime \prime}+p(z) w=0$ has two zeros $a, b$ in $\operatorname{Re}\{z\} \geqq 0$ with $|b| \geqq|a|$ and $|b|$ $\geqq|b-a|$. If $\arg (b-a)=\theta$, then by (7.6) we have

$$
\int_{0}^{R} \rho\left|p\left(a+\rho e^{i \theta}\right)\right| d \rho>1, \quad R=|b-a| .
$$

Now, let $r=|b|+\rho-R$; note that $r=|b|-|b-a|=\epsilon>0$ when $\rho=0$. Moreover,

$$
\begin{aligned}
\left|p\left(a+\rho e^{i \theta}\right)\right| & \leqq M\left(\left|a+\rho e^{i \theta}\right|\right) \\
& =M\left(\left|b-(R-\rho) e^{i \theta}\right|\right) \\
& \leqq M(|b|-R+\rho) \\
& =M(r) .
\end{aligned}
$$

Hence

$$
\int_{0}^{|b|} r M(r) d r \geqq \int_{\epsilon}^{|b|}(r-\epsilon) M(r) d r>1,
$$

contradicting (9.1), and proving the lemma.

This lemma leads to obvious zero-free regions and also gives us

THEOREM 9.1. Let $p(z)$ be analytic for $\operatorname{Re}\{z\} \geqq x_{0}$, and let $M(r)$ be defined as in Lemma 9.1. If $M(r)$ is monotone decreasing, and if 


$$
\int_{0}^{\infty} r M(r) d r \leqq 1,
$$

then no solution of $w^{\prime \prime}+p(z) w=0$ has more than 3 zeros in $\operatorname{Re}\{z\} \geqq x_{0}$.

Proof. Let $w(z)$ be any solution of $w^{\prime \prime}+p(z) w=0$, and suppose that $w(a)=0$, where $\operatorname{Re}\{a\} \geqq x_{0}$. According to the preceding lemma $w(z)$ cannot have another zero $z=b$ in that part of $\operatorname{Re}\{z\} \geqq x_{0}$ which lies outside the semicircle $\left|z-x_{0}\right|<\left|a-x_{0}\right|$ and which lies on the same side of the perpendicular bisector of the line joining $z=x_{0}$ and $z=a$ as $z=a$. In addition, $w(z)$ can have no zero in that part of the circle $|z-a|<\left|a-x_{0}\right|$ common to $\operatorname{Re}\{z\} \geqq x_{0}$. For, on interchanging the roles of $a$ and such a zero $b$, we see that $a$ would lie in the zero-free region corresponding to $z=b$.

In particular, then, we see that $w(z)$ can have no zeros in the part of $\operatorname{Re}\{z\} \geqq x_{0}$ common to a sector of angle $120^{\circ}$ with vertex at $z=x_{0}$ and having the line joining $z=x_{0}, z=a$ as axis of symmetry. The theorem now follows easily. For if a solution $w(z)$ had $n \geqq 4$ zeros, then for at least one pair of these zeros the angle subtended at $x_{0}$ by the lines joining them to $x_{0}$ would be less than or equal to $60^{\circ}$, which is impossible.

As mentioned in the introduction, a theorem of this type may be interpreted as a sufficient condition for 3-valence of the function $f(z)$ whose Schwarzian derivative is $2 p(z)$.

10. Zeros in a circle. In this section we use the Green's function to obtain a sufficient condition for disconjugacy in a circular region.

LEMMA 10.1. Let $p(z)$ be analytic in the circle $|z| \leqq R$, and suppose that a solution $w(z)$ of $w^{\prime \prime}+p(z) w=0$ has zeros at $a=R_{1} e^{-i \psi}, b=R_{2} e^{i \downarrow}, 0<\psi \leqq \pi / 2$. Then

$$
|b-a| \leqq 2 A \int_{0}^{A}(A-r) M(r) d r
$$

where

$$
M(r)=\max _{|z|=r}|p(z)|, \quad A=\max \left(R_{1}, R_{2}\right) \leqq R .
$$

Proof. By (7.3) we have

$$
1 \leqq \int_{a}^{b}|g(z, \zeta)||p(z)||d z|
$$

This time we take the path of integration to be the radial lines joining the points $z=a, z=b$ to the origin. Writing $\zeta=\rho e^{-i \psi}, z=r e^{ \pm i \psi}$, we have (if $\zeta$ is on the line segment joining 0 and $a$ ), 


$$
\begin{aligned}
1 \leqq & \int_{\rho}^{R_{1}} \frac{\left(R_{1}-r\right)\left(R_{2}^{2}+\rho^{2}-2 \rho R_{2} \cos 2 \psi\right)^{1 / 2}}{|b-a|} M(r) d r \\
& +\int_{0}^{\rho} \frac{\left(R_{1}-\rho\right)\left(R_{2}^{2}+r^{2}-2 r R_{2} \cos 2 \psi\right)^{1 / 2}}{|b-a|} M(r) d r \\
& +\int_{0}^{R_{2}} \frac{\left(R_{1}-\rho\right)\left(R_{2}-r\right)}{|b-a|} M(r) d r .
\end{aligned}
$$

We obtain a similar inequality with $R_{1}$ and $R_{2}$ interchanged if $\zeta$ is on the line segment joining 0 and $b$. In either case it is clear that the inequality is improved by replacing $R_{1}, R_{2}$ by $A$. With this change we now have

$$
\begin{aligned}
|b-a| \leqq & \int_{\rho}^{A}(A-r)\left(A^{2}+\rho^{2}-2 \rho A \cos 2 \psi\right)^{1 / 2} M(r) d r \\
& +\int_{0}^{\rho}(A-\rho)\left(A^{2}+r^{2}-2 r A \cos 2 \psi\right)^{1 / 2} M(r) d r \\
& +\int_{0}^{A}(A-\rho)(A-r) M(r) d r .
\end{aligned}
$$

Denoting the right side of this inequality by $f(\rho)$, we note that $f(A)=0$, while $f^{\prime}(\rho)<0$ for $\rho>0$, so that

$$
f(\rho) \leqq f(0)=2 A \int_{0}^{A}(A-r) M(r) d r,
$$

and the lemma is proved.

TheOREM 10.1. Let $p(z)$ be analytic in $|z| \leqq R$, and set

$$
M(r)=\max _{|z|=r}|p(z)|, \quad 0 \leqq r \leqq R .
$$

If

$$
R M^{1 / 2}(R) \int_{0}^{R}(R-r) M(r) d r \leqq \frac{\pi}{2},
$$

then the differential equation

$$
w^{\prime \prime}+p(z) w=0
$$

is disconjugate in the circle $|z|<R$.

Proof. Assume that the differential equation (1.2) has a solution $w(z)$ with two zeros in $|z|<R$. These zeros cannot be on the same radial line $z=r e^{i \omega}$. 
For, if they were, then by (7.6) we would have

$$
1<\int_{0}^{R}(R-r) M(r) d r
$$

this, together with (10.2), would imply $R^{2} M(R)<\pi^{2} / 4$. But according to Corollary 1.1, this is sufficient to insure the disconjugacy of (1.2) in $|z|<R$.

As in the proof of Theorem 2.1 there is no loss of generality in assuming these two zeros are $z_{1}=R_{1} e^{-i \psi}, z_{2}=R_{2} e^{i \psi}, 0<\psi \leqq \pi / 2$. By Lemma 10.1 we have

$$
2 A \int_{0}^{A}(A-r) M(r) d r \geqq\left|z_{1}-z_{2}\right|, A=\max \left(R_{1}, R_{2}\right) .
$$

In the circle $|z| \leqq A$, we have $|p(z)| \leqq M(A)$, hence by Corollary 1.1 it follows that $M(A) \geqq \pi^{2} /\left|z_{1}-z_{2}\right|^{2}$. Consequently

$$
2 A \int_{0}^{A}(A-r) M(r) d r \geqq \pi M^{-1 / 2}(A) .
$$

Since $R>A$, and $M(R) \geqq M(A)$, we have

$$
\int_{0}^{R}(R-r) M(r) d r>\int_{0}^{A}(A-r) M(r) d r \geqq \pi / 2 A M^{1 / 2}(A)>\pi / 2 R M^{1 / 2}(R) .
$$

This contradicts (10.2) and completes the proof of the theorem.

Note that the above theorem gives a new result only in the case that

$$
\int_{0}^{R}(R-r) M(r) d r<1 .
$$

For, if this integral is greater than or equal to unity, and (10.2) holds then, as noted in the proof,

$$
R^{2} M(R) \leqq \pi^{2} / 4,
$$

and the disconjugacy of (1.2) in $|z|<R$ follows from Nehari's theorem. The theorem cannot be sharp since (10.4) (which was used in the proof), is sharp only if $M(r)$, hence also $p(z)$, is constant, whereas one easily sees that Lemma 10.1 is not sharp for $p(z) \equiv$ constant. Nevertheless, (10.2) of ten provides a larger estimate for the radius of disconjugacy than (10.4). For example, in the case $w^{\prime \prime}+z^{2} w=0,(10.2)$ is satisfied if $R^{6} \leqq 6 \pi$, while (10.4) is satisfied if $R^{6} \leqq \pi^{3} / 8<6 \pi$.

\section{REFERENCES}

1. G. H. Hardy, J. E. Littlewood, and G. Polya, Inequalities, 2d ed., Cambridge University Press, 1952.

2. P. Hartman and A. Wintner, On an oscillation criterion of Liapounoff, Amer. J. Math. vol. 73 (1951) pp. 885-890. 
3. E. Hille, On the zeros of Sturm-Liouville functions, Arkiv. för Matematik, Astronomi och Fysik, vol. 16, no. 17, 1922.

4. - Oscillation theorems in the complex domain, Trans. Amer. Math. Soc. vol. 23 (1922) pp. 350-385.

5. - Non-oscillation theorems, Trans. Amer. Math. Soc. vol. 64 (1948) pp. 234-252.

6. - Remarks on a paper by Zeev Nehari, Bull. Amer. Math. Soc. vol. 55 (1949) pp. $552-553$.

7. Z. Nehari, The Schwarzian derivative and schlicht functions, Bull. Amer. Math. Soc. vol. 55 (1949) pp. 545-551.

8. - On the zeros of solutions of second-order linear differential equations, Amer. J. Math. vol. 76 (1954) pp. 689-697.

9. - Some criteria of univalence, Proc. Amer. Math. Soc. vol. 5 (1954) pp. 700-704.

10. - Univalent functions and linear differential equations, Lectures on Functions of a Complex Variable, University of Michigan Press, Ann Arbor, 1955, pp. 148-151.

11. V. V. Pokornyi, On some sufficient conditions for univalence, Doklady Akademii Nauk SSSR (N.S.) vol. 79 (1951) pp. 743-746.

12. B. Schwarz, Complex nonoscillation theorems and criteria of univalence, Trans. Amer. Math. Soc. vol. 80 (1955) pp. 159-186.

13. Choy-tak Taam, Oscillation theorems, Amer. J. Math. vol. 74 (1952) pp. 317-324.

14. Non-oscillation and comparison theorems of linear differential equations with complex-valued coefficients, Portugaliae Mathematica vol. 12 (1953) pp. 57-72.

15. - On the complex zeros of functions of Sturm-Liouville type, Pacific Journal of Mathematics vol. 3 (1953) pp. 837-843.

16. E. T. Whittaker and G. N. Watson, $A$ course of modern analysis, 4 th ed., Cambridge University Press, 1927.

17. A. Wintner, On the non-existence of conjugate points, Amer. J. Math. vol. 73 (1951) pp. 368-380.

18. - A criterion for non-oscillatory differential equations, Quarterly of Applied Mathematics vol. 7 (1949) pp. 115-117.

WASHINGTON UNIVERSITY, ST. Lours, Mo. 